\title{
Digitally Analyzed Conjunctival Redness: Does Repeated Conjunctival Provocation Intrinsically Cause Local Desensitization of the Eye?
}

\author{
Claas Gloistein Anatoli Astvatsatourov Silke Allekotte Ralph Mösges \\ Institute of Medical Statistics, Informatics and Epidemiology, Faculty of Medicine, University of Cologne, Cologne, \\ Germany
}

\section{Key Words \\ Conjunctival provocation $\cdot$ Rhinoconjunctivitis · Placebo treatment $\cdot$ Conjunctival hyperemia $\cdot$ Objective allergy symptom · Digital analysis}

\begin{abstract}
Background: Allergic reactions in patients with seasonal or perennial rhinoconjunctivitis mediated by airborne allergens can be effectively assessed with the conjunctival provocation test (CPT). The CPT is a fast and easy diagnostic procedure that challenges the ocular mucosa with instillations of allergen solutions into the conjunctival region. This paper aimed to investigate the possible influence of repeated diagnostic CPT procedures on the patient's clinical presentation, i.e. to analyze desensitization effects caused by diagnostic solutions and to show the reproducibility of CPT results. Methods: Treatment progress in 120 placebo-treated patients from 2 immunotherapeutic dose-finding studies was estimated and documented, as based on the CPT which was applied at 4 visits with intervals of 4,8 and 16 weeks. High-resolution digital photos collected as part of the CPT documentation were analyzed by an external observer and by digital analysis software to determine conjunctival redness, completely independent of the subjectivity of investigators and patients. Results: Two extremal scenarios of the redness changes were considered after provocation with 10,000 standard quality units $/ \mathrm{ml}$. A
\end{abstract}

maximal decrease of about $3 \%$ (t test: $p=0.0002 ; \mathrm{U}$ test: $p=$ 0.001 ) and a minimal decrease of about $1 \%$ (t test: $p=0.254$; $U$ test: $p=0.431$ ) were found. Conclusions: The observed decrease in conjunctival hyperemia can be explained by local desensitization or by placebo effect. Due to the setup of both studies considered, we could not ascertain how these factors influence the decrease in redness. In order to attribute the observed effects to local conjunctival desensitization with certainty, further pilot studies are needed.

두 2016 S. Karger AG, Base

\section{Introduction}

Ocular symptoms in allergies are not only common, but also distressing for sufferers. According to the World Allergy Organization estimation [1], between 20 and $40 \%$ of the world population are affected by allergies. In the recent 'Allergies in America' survey, $>50 \%$ of nasal allergy patients stated that tearing and $\mathrm{red} / \mathrm{itching}$ eyes were moderately to extremely bothersome [2-4]. For about of $15 \%$ of all nasal allergy sufferers, the ocular component of allergic hypersensitivity reactions was the most bothersome symptom. Furthermore, allergic symptoms significantly impair social functioning and quality of life [5], and sufferers score lower on the Visual Functioning Questionnaire 25 (VQF-25) [6, 7]. Nevertheless, the ocu-

\section{KARGER 125}

(c) 2016 S. Karger AG, Base

$1018-2438 / 16 / 1684-0277 \$ 39.50 / 0$

E-Mail karger@karger.com

www.karger.com/iaa
Correspondence to: Dr. Claas Gloistein, Dr. Ralph Mösges

Institute of Medical Statistics, Informatics and Epidemiology, Faculty of Medicine University of Cologne, Lindenburger Allee 42

DE-50931 Cologne (Germany)

E-Mail cgloiste@ smail.uni-koeln.de, ralph.moesges@uni-koeln.de 
lar components of allergies are underdiagnosed and remain widely unconsidered [8].

In this work, we investigated the ocular symptoms in placebo-treated patients from 2 immunotherapeutic dose-finding studies. Patients showed a positive reaction in the skin-prick test (SPT) with a wheal diameter $>3 \mathrm{~mm}$, a negative control $<2 \mathrm{~mm}$ and positive specific IgE reactivity to the allergen $(>0.7 \mathrm{kU} / \mathrm{l})$. The primary diagnosis of allergic disease was based on the SPT and specific IgE blood test. These methods are well established in daily clinical routine, applied easily, and can diagnose the immune system's sensitization to a specific allergen. However, besides being imprecise, they are often not suitable for evaluating the severity of an allergic reaction $[9,10]$ and do not necessarily provide information on how to interpret the results clinically. Neither nasal nor ocular symptoms are captured this way.

The conjunctival provocation test $(\mathrm{CPT})$ represents a sensitive method used to screen for airborne allergies, as it addresses well-assessable allergic symptoms and the severity of hypersensitivity reactions directly at the involved organ, i.e. the provoked mucosa of the eyes. The CPT method is used in clinical practice as well as for the documentation of treatment progress in some immunotherapeutic dose-finding studies. Furthermore, it can detect occupational allergies [11] and exclude clinically silent presentations in cases where clinical findings contradict the patient's anamnesis [12]. The CPT is accredited by regulatory authorities for measuring the allergic response in seasonal and perennial allergic rhinitis and rhinoconjunctivitis [13].

The advantages of the CPT are manifold. The method is easy to perform, low-priced, rarely elicits systemic reactions and is also independent of the examiner's experience and the patient's compliance $[10,11,14,15]$. The CPT makes it possible to assess the therapeutic (pharmacological) effect on the severity of allergic reactions [16], determine optimal treatment doses in immunotherapeutic dose-finding studies and define the current status of a patient's allergic severity. For patients with pronounced conjunctivitis, one can objectify the treatment results or the current allergy status using digital image processing when the reddening of the challenged eyes is documented with high-resolution photos. An additional poststudy analysis of eye redness before and after provocation complements the patient's subjective symptom scores and the investigator's rating of the ocular reactions.

Since the first trials on prophylactic inoculation against hay fever were published by Noon [17] in 1911, it has been known that specific immunotherapy may alter the course of an allergic reaction. Immunotherapy aims at either systemic or local desensitization. The corresponding immunotherapeutic medications usually contain modified allergens or parts of allergens and can be administered via different routes, e.g. sublingual, subcutaneous or directly to the affected mucosal area. The underlying principle of these therapies is usually the administration of gradually increasing doses of allergens, changing the way how the immune system reacts over the treatment time which may last up to 3 years.

With the CPT procedure, several allergen provocations are conducted in order to elicit an allergic response to the allergen solution at a certain concentration. These solutions are applied in increasing concentrations before, during or after immunotherapeutic intervention to assess treatment progress. The CPT and immunotherapy both involve repeated exposure to allergenic substances. Thus, the question has arisen as to whether the CPT itself as a diagnostic procedure might elicit local immunotherapeutic effects. This diagnostic tool could influence immunotherapeutic study results, i.e. medication efficacy analyzed in dose-finding studies. In other words, if the CPT induces an immunotherapeutic effect, it will cause a baseline shift of conjunctival hyperemia. This potentially unconsidered shift could enhance the visible results as it adds to the medication effect, meaning that the real therapeutic effect would be smaller than estimated.

The desensitization influence of CPT diagnostics can be effectively investigated in patients receiving placebo in appropriate clinical desensitization studies. The aim of this paper was to show the influence of the CPT on the outcomes of placebo group patients. Data from 2 doubleblind, placebo-controlled, randomized immunotherapeutic trials on repeated conjunctival allergen challenge were used. To fulfill the aim of this work, the results of multiple allergen challenges were compared, in order to answer the question whether the outcomes of these challenges are stable, i.e. with respect to the allergen threshold level or symptom severity. The treatment outcome itself was not subject to evaluation in this work; only placebo group patients were selected for our analysis.

\section{Material and Methods}

\section{Patients and Data Collection}

In total, 295 patients aged 18-75 years were included in 2 prospective, double-blind, randomized, placebo-controlled, multicenter, dose-finding studies with sublingual immunotherapy (SLIT). Both studies had been approved by the corresponding Ethics Committees and by an agency of the German Federal Ministry 
of Health (Paul Ehrlich Institute). Both clinical trials were carried out from July to September 2013 (EudraCT No. 2013-000617-20 and No. 2013-002129-43). All patients gave written consent before being enrolled.

The CPT was performed at 4 different visits over a total of 14 16 weeks for the house-dust mite study and 18-19 weeks for the birch-alder pollen study. Only patients who had suffered for at least 2 years from the corresponding allergy prior to study inclusion and who had positive SPT and sIgE findings were included. Of the 120 patients who received placebo, 24 were allergic to house-dust mite and 96 to birch-alder pollen.

\section{CPT Procedure}

Patients were challenged with standardized allergen extract ALK-lyophilized SQ (ALK-Abelló A/S, Hørsholm, Denmark) according to the following schedule: visit 0 (V0) at the screening of patients, examination of ocular reaction after provocation and determination of the threshold dosage, visit 1 (V1) at 3-28 days after V0, prior to sublingual immunotherapy (SLIT), visit 3 (V3) at 28 \pm 7 days after treatment start at V1 and visit 4 (V4) at $84 \pm 7$ days after treatment start at V1 for the study with house-dust mites, and at around 120 days after V1 for the study with birch-alder pollen.

At each visit, a single drop of the allergen solution was applied to the lower conjunctival sac in consecutive dosages of $100,1,000$ and 10,000 standard quality units (SQ-U)/ml, according to the CPT procedure introduced by Riechelmann et al. [15], with the amendment of adding a lower dose of $100 \mathrm{SQ}-\mathrm{U} / \mathrm{ml}$. By definition, only the left eye was provoked with allergen solution; the right eye received the allergen-free solution ALK diluent at each visit, serving as a blank value to screen for false-positive reactions (unilateral CPT). After waiting $10 \mathrm{~min}$, the local investigator documented the patient's reaction and proceeded with the next higher dosage if the ocular reaction was negative. In the case of a positive reaction, the provocation test was stopped at this point and documented as previously described [7].

\section{Technical Equipment for Photographic Documentation}

Before starting the CPT and after each subsequent step, a local investigator photographed the patient's ocular reaction. Centers were therefore equipped with a special stand which fixed a camera and macro LED light in front of the patient's eye. To keep the eye in a steady position and obtain clear images, a chin and forehead rest stabilized the patient's head. The camera rail slides enabled the investigator to position the camera with respect to the fixed eye in all spatial directions.

A digital camera suitable for macrophotography was used which produced JPEG images of approximately 3.6 MB each with a resolution of $3,648 \times 2,736 \mathrm{dpi}$. The images were saved on SD cards and then copied onto a data storage disk. The investigators were instructed to use the following camera parameters: autofocus with the focal point positioned on the conjunctiva below the iris, white balance of $4,500 \mathrm{~K}$, flash switched off, photosensitivity set to ISO 800 , a small focal aperture of $\mathrm{f} / 10$ and an automatic shutter speed adapted to the aperture. In order to uncover and record the maximal area of the conjunctival region, patients were asked to pull down their lower eyelid.

\section{Investigator's Rating}

The CPT procedures for both studies considered in this work were conducted in 24 study centers in Germany, mostly by oto-
Table 1. The Gronemeyer grading scale to assess allergic severity in $\mathrm{CPT}$ [11]

\begin{tabular}{ll}
\hline Stage & Findings \\
\hline 0 & $\begin{array}{l}\text { No subjective or visible reaction } \\
\text { I }\end{array}$ \\
II & $\begin{array}{l}\text { Stage I plus tearing, vasodilation of the conjunctiva } \\
\text { bulbi } \\
\text { Stage II plus vasodilation and erythema of the con- }\end{array}$ \\
III & $\begin{array}{l}\text { junctiva tarsi, blepharospasm } \\
\text { Stage III plus chemosis, lid swelling }\end{array}$ \\
IV &
\end{tabular}

The threshold value for a positive CPT is stage II.

Table 2. Number of analyzed patients per visit and different concentrations of allergen solution used for the CPT

\begin{tabular}{|c|c|c|c|c|}
\hline \multirow[t]{2}{*}{ Concentration } & \multicolumn{4}{|c|}{ Patients, $\mathrm{n}$} \\
\hline & at $\mathrm{V} 0$ & at V1 & at V3 & at V4 \\
\hline $0 \mathrm{SQ}-\mathrm{U} / \mathrm{ml}$ & 110 & 110 & 97 & 104 \\
\hline 100 SQ-U/ml & 111 & 117 & 102 & 111 \\
\hline $1,000 \mathrm{SQ}-\mathrm{U} / \mathrm{ml}$ & 106 & 104 & 97 & 107 \\
\hline $10,000 \mathrm{SQ}-\mathrm{U} / \mathrm{ml}$ & 87 & 85 & 84 & 96 \\
\hline
\end{tabular}

rhinolaryngologists or dermatologists specializing in allergies. The investigators assessed and documented ocular reactions according to the Gronemeyer scale [11] presented in table 1. Each allergic reaction stage consisted of the objective symptoms (i.e. visible) of reddening, tearing and, if detectable, swelling, and the subjective symptoms (i.e. perceived by the patient) of itching and foreign-body sensation. Stages 0 and I were considered negative results, as the visible vasodilatation defined from stage II onward was needed to objectify the severity of symptoms on a digital basis. The threshold allergic severity at which the CPT was discontinued was defined as stage II according to the Gronemeyer scale, i.e. if a patient's reaction reached stage II, the local investigator stopped the CPT. Some patients were challenged once with the lowest concentration of 100 SQ-U/ml, or once with 100 SQ-U/ml and once with 1,000 SQ-U/ml, and others were challenged with each of the 3 concentrations (100, 1,000 and 10,000 SQ-U/ml). Thus, the patients each underwent different numbers of provocations and therefore had different numbers of digital images that were assessed by the investigators based on the Gronemeyer scale. Table 2 shows the number of collected conjunctival pictures per visit and concentration. The number of pictures also varied between visits. For instance, if a patient reached stage II after provocation with $1,000 \mathrm{SQ} \mathrm{U} / \mathrm{ml}$ at 1 visit, he/she could reach stage II with 100 or $10,000 \mathrm{SQ}-\mathrm{U} / \mathrm{ml}$ at the next visit. 


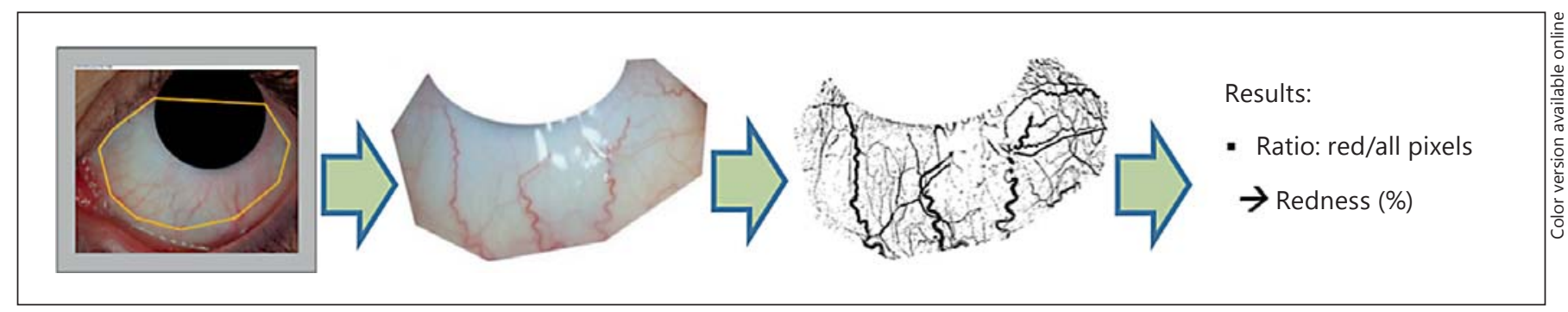

Fig. 1. Data analysis steps. Selection of conjunctival ROI, vessel detection via application of ACE algorithm and calculation of redness.

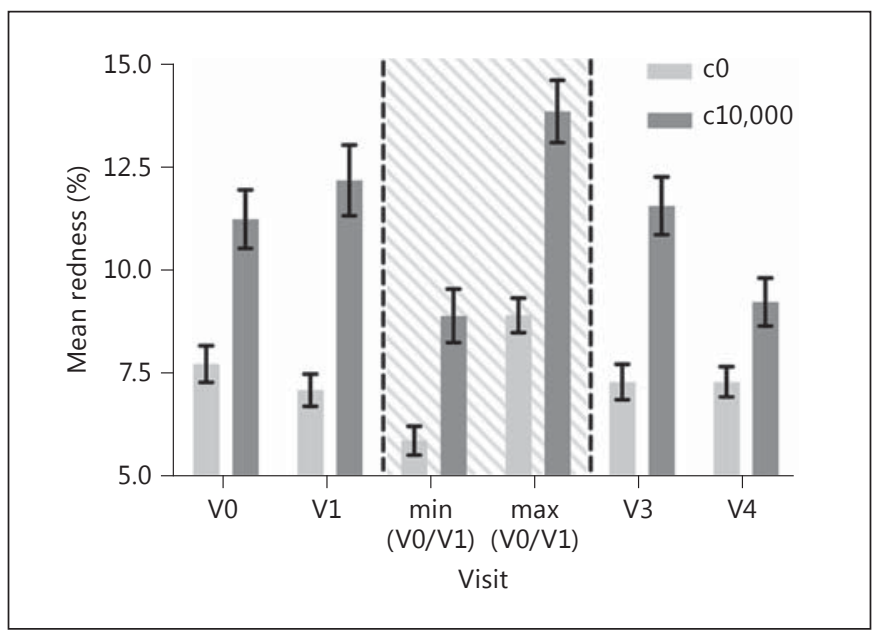

Fig. 2. Average conjunctival redness over the course of visits before any provocation and after provocation with 10,000 SQ-U/ml. Data points are calculated as mean values of all patients; error bars represent SEM.

\section{Digital Analysis}

The aim of the digital image analysis was to determine redness from the conjunctival macrophotographs.

Using the Olympus software package cell^ $\mathrm{F}$, Dogan et al. [12] showed digital analysis to be a suitable and sensitive method for quantifying ocular allergic reactions. The cell^ $\mathrm{F}$ filters applied to the grayscale images of the conjunctiva were successfully used in a previous CPT study [12] and to detect neoangiogenesis in corneal transplants by Bock et al. [18].

We conducted the conjunctival redness analysis using the Matlab package and by applying the adaptive contrast enhancement (ACE) algorithm [19]. Figure 1 shows the workflow of the data analysis. In order to evaluate the conjunctival reddening induced by CPT, the photographs were prepared as follows. (1) The conjunctival region of interest (ROI) to be analyzed was chosen manually using NIH ImageJ software (the iris and eyelids were excluded). As the bulbar position may have varied slightly in the images of one CPT series [20], it was important to display all pictures from 1 visit simultaneously, in order to determine the conjunctival in- tersection set visible in all of them. (2) After performing white balance, the ACE algorithm was applied to identify conjunctival vessels. ACE thereby compared the intensity value of an individual pixel with the arithmetic mean intensity of the neighboring pixels belonging to a cluster around the examined pixel. Afterwards, the images were binarized and background noise was removed. (3) The result of the digital image analysis was the ratio of red pixels/ all pixels, giving a percentage value of redness.

Figure 2 shows the development of the mean conjunctival redness over the course of visits before any provocation (initial conditions) and after provocation with 10,000 SQ-U/ml. Error bars represent the SEM. As expected, the initial conditions were stable, i.e. there was no considerable mean difference in the redness of the unprovoked eyes between the visits. All values remained between 7.13 and $7.75 \%$. After the CPT with 10,000 SQ-U/ml, a distinct increase of up to $12.15 \%$ was observed, meaning a relative increase in redness of about $70 \%$. Comparison of the baseline visits (V0 and $\mathrm{V} 1$ ) with the last visit (V4) reveals that this increase of redness after provocation was considerably smaller at the end of the study than at the beginning.

\section{Results}

Figure 3 illustrates the conjunctival redness gradient over the course of the visits. The change in redness is the difference of the conjunctival redness before and after provocation for each patient. The redness before provocation is the baseline redness, i.e. an SQ-U/ml concentration of zero ( $\mathrm{c} 0$ ). The calculated redness values for provocations with $100,1,000$ and $10,000 \mathrm{SQ}-\mathrm{U} / \mathrm{ml}$ are denoted as 'c100', 'c1,000' and 'c10,000', respectively. Values were averaged and error bars represent the standard error of the mean (SEM), which is equal to the standard deviation divided by the square root of the number of subjects in the corresponding provocation group. V0 and V1 are the baseline visits, which took place before therapy start with either active treatment or placebo. CPT challenges with instillations of 100 SQ-U/ml and 1,000 SQ-U/ml elicited no large changes - the redness values remained close to 


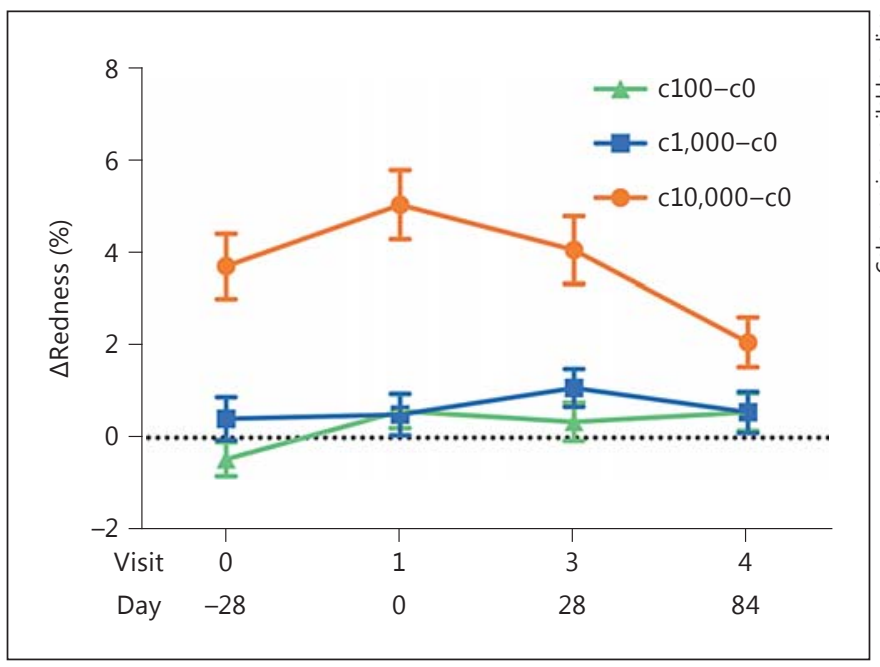

Fig. 3. Development of conjunctival redness over the course of the visits according to the dose of CPT solution. Data points are calculated as the mean values of all patients; error bars represent SEM. $\Delta$ Redness $=$ Change in redness .

zero. After instillation of $10,000 \mathrm{SQ}-\mathrm{U} / \mathrm{ml}$, the redness value rose at V1 and decreased thereafter at V3 and V4. The Mann-Whitney U test and the Student $t$ test showed a nonsignificant difference between redness measured at $\mathrm{V} 0$ and at V4. However, between V1 and V4, the CPT with 10,000 SQ-U/ml showed a distinct decrease in redness with a $\mathrm{p}$ value of $0.3 \%$. The $\mathrm{p}$ values of both applied tests are presented in table 3.

At the baseline visits, V0 and V1, one also notices a tangible spread in redness values (fig. 3). To estimate the greatest and least possible difference in redness over the course of the visits, 2 virtual data points were introduced, i.e. minimal and maximal redness values at $\mathrm{V} 0$ and $\mathrm{V} 1$ : $\min (\mathrm{V} 0 / \mathrm{V} 1)$ and $\max (\mathrm{V} 0 / \mathrm{V} 1)$. They were calculated for each patient and subsequently averaged. Thus, the minimal and maximal values of the baseline visits represent 2 extreme baseline reaction scenarios.

Figure 4 shows the development of redness after provocation with 10,000 SQ-U/ml over the course of the visits. Here, in addition to the information shown in figure 3, $\min (\mathrm{V} 0 / \mathrm{V} 1)$ and $\max (\mathrm{V} 0 / \mathrm{V} 1)$ are added to the plot inside the hatched area. Linear regression is added to illustrate the possible minimal (blue) and possible maximal (red) decrease in redness. Figure 5 shows all values grouped by provocation dose (fig. 3,4 ).

Between V0 and V4, the results showed a nonsignificant reduction in allergic conjunctival redness of $1.63 \%$ (paired test: $\mathrm{p}=0.116$; paired $\mathrm{U}$ test: $\mathrm{p}=0.244$ ), but be-

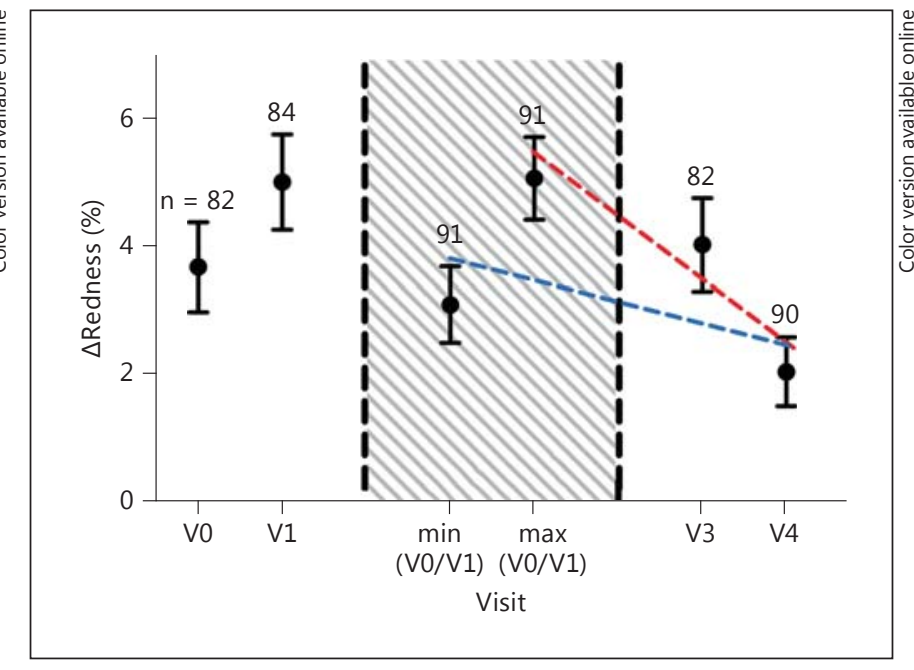

Fig. 4. Development of conjunctival redness after provocation with 10,000 SQ-U/ml over the course of the visits. Calculated data points $\min (\mathrm{V} 0 / \mathrm{V} 1), \max (\mathrm{V} 0 / \mathrm{V} 1)$ and best fit line added. Error bars represent SEM. $\Delta$ Redness $=$ Change in redness.

Table 3. Change in redness at 10,000 SQ-U/ml

\begin{tabular}{llll}
\hline Visit interval & $\begin{array}{l}\text { Difference of } \\
\text { means } \\
\end{array}$ & p value & \\
\cline { 3 - 4 } & $(\Delta \mu$ in $\%)$ & U test & t test \\
\hline V0-V1 & -1.330 & 0.146 & 0.136 \\
V0-V3 & -0.353 & 0.385 & 0.599 \\
V0-V4 & 1.630 & 0.244 & 0.116 \\
V1-V3 & 0.977 & 0.468 & 0.520 \\
V1-V4 & 2.960 & 0.004 & 0.003 \\
V3-V4 & 1.983 & 0.020 & 0.109 \\
$\min (V 0 / V 1)-V 3$ & -0.936 & 0.282 & 0.318 \\
$\min (V 0 / V 1)-V 4$ & 1.047 & 0.431 & 0.254 \\
$\max (\mathrm{V} 0 / \mathrm{V} 1)-\mathrm{V} 3$ & 1.037 & 0.343 & 0.404 \\
$\max (\mathrm{V} 0 / \mathrm{V} 1)-\mathrm{V} 4$ & 3.020 & 0.001 & 0.000 \\
\hline
\end{tabular}

Calculated $\mathrm{p}$ values using the parametric Student $\mathrm{t}$ test and nonparametric Mann-Whitney U test between all visits for 10,000 $\mathrm{SQ}-\mathrm{U} / \mathrm{ml}$.

tween V1 and V4, the reduction in redness of $2.96 \%$ (paired t test: $\mathrm{p}=0.003$; paired $\mathrm{U}$ test: $\mathrm{p}=0.004$ ) was significant. With respect to the difference between $\max (\mathrm{V} 0 /$ V1) and V4, a significant reduction in redness was observed (3.02\%; t test: $\mathrm{p}=0.0002$; $\mathrm{U}$ test: $\mathrm{p}=0.001)$. For the $\min (\mathrm{V} 0 / \mathrm{V} 1)$ to $\mathrm{V} 4$ scenario, however, no significance for the reduction in redness was found $(1.05 \%$; $t$ test: $\mathrm{p}=$ 0.254; $U$ test: $\mathrm{p}=0.431$ ). 
Fig. 5. Conjunctival redness means grouped by CPT dose. Data points are calculated as mean values of all patients; error bars represent SEM. Two calculated data points are added: $\min (\mathrm{V} 0 / \mathrm{V} 1)$ and $\max (\mathrm{V} 0 / \mathrm{V} 1)$. $\Delta$ Redness $=$ Change in redness.

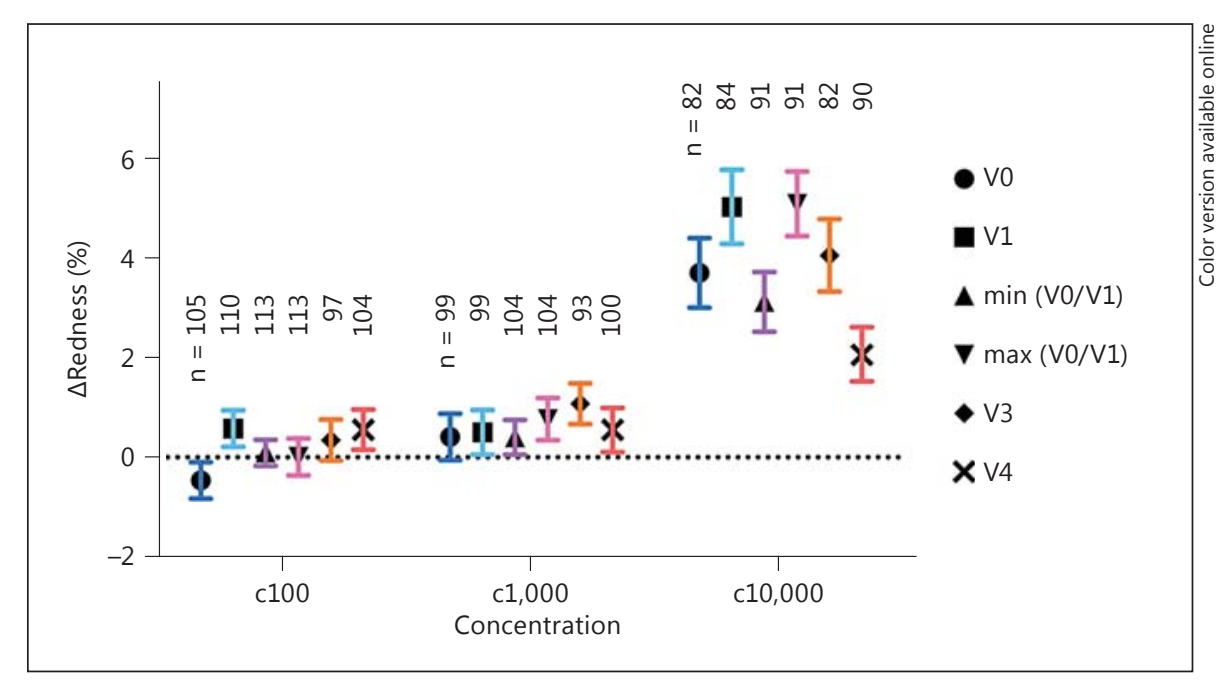

\section{Discussion}

This work analyzed the placebo groups of 2 SLIT studies for dust-mite and birch-pollen allergies, using CPT to document treatment outcome. The following steps are discussed below: (1) the gradient application of 3 different allergen concentrations, 100, 1,000 and 10,000 SQ-U/ $\mathrm{ml}$, administered in the lower conjunctival sac, and (2) the subsequent digital image processing of the conjunctival ROI, including manual segmentation and determination of redness with the vessel finder written in Matlab. It is important that here the redness value was defined as the percentage of red pixels in the ROI, i.e. as the ratio of the detected red pixels/total number of pixels in the ROI. This ratio was the digital analysis outcome, which estimated redness independently of the investigator's or observer's ratings and allowed an objective comparison of changes at a single visit as well as a comparison between different visits. Thus, the redness value provided an objective parameter of the severity of allergic reactions.

Overall results showed a nearly constant degree of redness over the course of visits after provocation with 100 $\mathrm{SQ}-\mathrm{U} / \mathrm{ml}$ and 1,000 SQ-U/ml, whereas after application of $10,000 \mathrm{SQ}-\mathrm{U} / \mathrm{ml}$, a distinct decrease in redness could be identified. Nonreaction after CPT may have several explanations. One explanation might be that the chosen concentrations were too low to elicit detectable hyperemia in the ROI. The allergic potential of dust-mite allergens, for example, might be less than other allergens, meaning that the solution gradient for dust mites should have started at a higher concentration. The optimal starting value would therefore have been $300-2,000$ SQ-U/ml, subsequently increasing to $30,000-100,000 \mathrm{SQ}-\mathrm{U} / \mathrm{ml}$. It is difficult to find reliable data on this point in the literature. So far, allergen concentrations have not yet been completely standardized, although attempts to do so have been made in the past. Allergen concentrations continue to differ from manufacturer to manufacturer, and the substances themselves vary in allergic potency, making them difficult to compare.

Another reason why patients did not visibly react to low-concentration solutions might be differences in the individual retention time of the allergens within the patient population. The 10 -min waiting period between the allergen application and reaction assessment was required by the protocols of both studies. Thus, a comparatively strong reaction at 10,000 SQ-U/ml might actually have been caused by the long retention time of allergens from the previous provocations. A very interesting point for further studies would be to investigate the retention time distribution and its influence on the redness value.

At V0 and V1, a nonsignificant change in redness between provocations with 100 and 1,000 SQ-U/ml was observed (fig. 3, 5). This finding may be due to technical reasons such as watery eyes, variations in camera focus and considerably different eye positions. Such measurement uncertainties influence the determined redness and could account for redness that was calculated but not actually visible in provocations with 100 and 1,000 SQ-U/ $\mathrm{ml}$. The baseline pictures had been taken before any solutions were applied. Some of the patients presented with rather dry eyes and a low level of tear film. Thus, conjunctival blood vessels were clearly pictured and could be properly addressed in the subsequent digital analysis. 
Dropping allergen solution in the conjunctival sac temporarily increased the amount of tear film by adding fluid. It also stimulated tear film production by generating a foreign-body sensation. Pictures of the watery eyes turned out to be more difficult to analyze and could be an additional source of measurement uncertainty. New light reflexes appeared and vessels were often less sharp in the pictures. The analysis software attempted to take these effects into account but may have ended up excluding parts of the ROI if the light reflexes were too bright, thereby concealing useful information. Therefore, a challenged eye may have been calculated as less red than the unchallenged eye at baseline.

With respect to CPT using 10,000 SQ-U/ml, distinct changes in conjunctival redness could be observed. The change in redness exhibited a maximum at the baseline visit V1. The differences between V0 and V1 were not significant. Factors influencing conjunctival redness could have included changing atmospheric parameters (temperature, humidity, etc.) and the patient's immunological or emotional status between the baseline visits.

Over the course of both studies, the redness at 10,000 SQ-U/ml constantly decreased up to the last visit. Even though patients did not receive an active ingredient, a possible placebo effect may have been a component of the detected decrease at V3 and V4. With V0 and V1 being equivalent baseline visits, the question arose as to which of these visits should be used to compare the outcome at the end of the studies. We therefore introduced a virtual visit, choosing either minimum or maximum values to create a new dataset. This allowed the evaluation of minimal and maximal changes in redness, respectively. Again, when this new value was compared to minimal redness, min (V0/V1), at the beginning of the studies, no significant change to V4 could be identified. A comparison to the maximal baseline value, $\max (\mathrm{V} 0 / \mathrm{V} 1)$, however, revealed a significant decrease in redness ( $t$ test: $p=0.0002$; $\mathrm{U}$ test: $\mathrm{p}=0.001)$. In this work, however, a possible placebo effect cannot be ruled out, as patients were randomized into placebo groups after V1.

Results from several studies conducting local conjunctival immunotherapy suggest that local conjunctival desensitization in general is indeed possible. Machida et al. [21] sensitized a group of guinea pigs with an injection of ovalbumin. After a latency period of 3 weeks, 1 eyedrop of low-concentration $(10 \mu \mathrm{g} / \mathrm{ml})$ ovalbumin was administered daily over a course of 3 weeks. A subsequent provocation with $20 \mathrm{mg} / \mathrm{ml}$ of ovalbumin eyedrops followed after 6 weeks total time and was compared to a control group. There was no change in the conjunctival clinical score in the early-phase reaction 30 min after allergen challenge, but an investigation showed a decrease in latephase reaction symptoms. The total number of inflammatory cells and the number of eosinophils in the treated group at 8 and $24 \mathrm{~h}$, respectively, after the provocation, were significantly lower than those in the control group.

Del Prete et al. [22] analyzed a group of 40 patients who suffered from seasonal allergic conjunctivitis triggered by different allergens (Dermatophagoides, pollen and fungus). Half of the patients received eyedrops in both eyes as local ocular immunotherapy. A diluted solution of $10 \%$ of the allergen concentration was administered to provoke a 3-mm-diameter wheal in an SPT. Clinical symptom scores, subjective symptoms and cytology improved significantly in the treated group after 1 year of treatment. Different allergens were used in this study, suggesting this method may be suitable for any kind of allergen.

Pfaar et al. [23] conducted a pilot study, using titrated CPT without immunotherapy in 23 adult patients who were allergic to airborne pollen, to estimate the severity of the allergic response. Measurements were carried out several times in weekly intervals to determine the testretest correlation and possible deviations. Considering the error of measurement and influence of environmental factors, their results suggest local mucosal desensitization by CPT.

The present investigation showed a decrease in conjunctival hyperemia in placebo group patients who underwent CPT. The CPT results obtained at 2 visits before placebo treatment were compared with the $\mathrm{CPT}$ results after the treatment period.

Two extreme scenarios were also considered to determine maximal and minimal decrease in redness over the course of 2 studies after conjunctival provocation with $10,000 \mathrm{SQ}-\mathrm{U} / \mathrm{ml}$. A maximal decrease in conjunctival redness of about $3 \%$ ( $t$ test: $p=0.003$, $U$ test: $p=0.004$ ) and a minimal decrease in redness of about $1 \%$ ( $\mathrm{t}$ test: $p=0.116$, $U$ test: $p=0.244$ ) were found.

The revealed significance between $\max (\mathrm{V} 0 / \mathrm{V} 1)$ and $\mathrm{V} 4$ redness may indicate one of two possibilities, a clear placebo effect and/or an actual local mucosal desensitization effect induced by the CPT procedure. Due to the setup of both therapy studies, we could not ascertain which of these factors was responsible for the decrease in redness. To be able to attribute the effects observed here to local conjunctival desensitization or to placebo effect with certainty, further pilot studies are needed that do not implement either active treatment or placebo. 
In conclusion, an observed decrease in redness over the course of a study might actually be a combination of both desensitization and a placebo effect. Thus, to avoid an overestimation of the therapeutic effect observed in actively treated patients, the therapy effect should be considered positive if the decrease in symptom severity of the treatment group is significantly higher than that of the placebo group.

\section{Acknowledgments}

The authors express their deep thanks to Mrs. Gena Kittel for her valuable comments and editorial assistance in finalising this manuscript.

\section{References}

1 Pawankar R CG, Holgate ST, Lockey RF: WAO White Book on Allergy. Milwaukee, World Allergy Organization, 2011.

2 Healthstar Communications: Allergies in America: executive summary. www.myallergiesinamerica.com (2007).

3 Bousquet PJ, Demoly P, Devillier P, Mesbah $\mathrm{K}$, Bousquet J: Impact of allergic rhinitis symptoms on quality of life in primary care. Int Arch Allergy Immunol 2013;160:393-400.

4 Lightman S, Scadding GK: Should intranasal corticosteroids be used for the treatment of ocular symptoms of allergic rhinoconjunctivitis? A review of their efficacy and safety profile. Int Arch Allergy Immunol 2012;158:317325.

5 Scadding GK, Durham SR, Mirakian R, Jones NS, Leech SC, Farooque S, Ryan D, Walker SM, Clark AT, Dixon TA, Jolles SR, Siddique N, Cullinan P, Howarth PH, Nasser SM: BSACI guidelines for the management of allergic and non-allergic rhinitis. Clin Exp Allergy 2008;38:19-42.

6 Pitt AD, Smith AF, Lindsell L, Voon LW, Rose PW, Bron AJ: Economic and quality-of-life impact of seasonal allergic conjunctivitis in Oxfordshire. Ophthalmic Epidemiol 2004;11: 17-33.

7 Smith AF, Pitt AD, Rodruiguez AE, Alio JL, Marti N, Teus M, Guillen S, Bataille L, Barnes JR: The economic and quality of life impact of seasonal allergic conjunctivitis in a Spanish setting. Ophthalmic Epidemiol 2005;12:233-242.

8 Brozek JL, Bousquet J, Baena-Cagnani CE, Bonini S, Canonica GW, Casale TB, van Wijk RG, Ohta K, Zuberbier T, Schunemann HJ: Allergic Rhinitis and its Impact on Asthma (ARIA) guidelines, 2010 revision. J Allergy Clin Immunol 2010;126:466-476.
9 van Hage-Hamsten M, Pauli G: Provocation testing with recombinant allergens. Methods 2004;32:281-291.

10 Riechelmann H, Epple B, Gropper G: Comparison of conjunctival and nasal provocation test in allergic rhinitis to house dust mite. Int Arch Allergy Immunol 2003;130:51-59.

11 Chelminska $M$, Niedoszytko $M$, Jassem E: Clinical value of conjunctival allergen challenge in diagnosing allergic conjunctivitis related to latex. Int Arch Allergy Immunol 2011; 154:149-154.

12 Dogan S, Astvatsatourov A, Deserno TM, Bock F, Shah-Hosseini K, Michels A, Mosges R: Objectifying the conjunctival provocation test: photography-based rating and digital analysis. Int Arch Allergy Immunol 2014;163: 59-68.

13 Godicke V, Hundt F: Registration trials for specific immunotherapy in Europe: advanced guidance from the new European Medical Agency guideline. Allergy 2010;65:1499-1505.

14 Horak F, Bergera U, Menapace R, Schuster N: Quantification of conjunctival vascular reaction by digital imaging. J Allergy Clin Immunol 1996;98:495-500.

15 Riechelmann H, Bachert C, Goldschmidt O, Hauswald B, Klimek L, Schlenter WW, Tasman AJ, Wagenmann M: Application of the nasal provocation test on diseases of the upper airways. Position paper of the German Society for Allergology and Clinical Immunology (ENT Section) in cooperation with the Working Team for Clinical Immunology (article in German). Laryngorhinootologie 2003;82:183-188.
16 Ciprandi G, Buscaglia S, Marchesi E, Danzig M, Cuss F, Canonica GW: Protective effect of loratadine on late phase reaction induced by conjunctival provocation test. Int Arch Allergy Immunol 1993;100:185-189.

17 Noon L: Prophylactic inoculation against hay fever. Lancet 1911;177:1572-1573.

18 Bock F, Matthaei M, Reinhard T, Böhringer D, Christoph J, Ganslandt T, Cursiefen C: High-dose subconjunctival cyclosporine a implants do not affect corneal neovascularization after high-risk keratoplasty. Ophthalmology 2014;121:1677-1682.

19 Wu D, Zhang M, Liu JC, Bauman W: On the adaptive detection of blood vessels in retinal images. IEEE Trans Biomed Eng 2006;53:341343.

20 Sarandi I, Classen DP, Astvatsatourov A, Pfaar O, Klimek L, Mosges R, Deserno TM: Quantitative conjunctival provocation test for controlled clinical trials. Methods Inf Med 2014;53:238-244.

21 Machida H, Nakagami T, Watanabe I: Local ocular immunotherapy for experimental allergic conjunctivitis. Jap J Ophthalmol 2000; 44:634-638.

22 Del Prete A, Loffredo C, Carderopoli A, Caparello O, Verde R, Sebastiani A: Local specific immunotherapy in allergic conjunctivitis. Acta Ophthalmol 1994;72:631-634.

23 Pfaar O, Claßen DP, Astvatsatourov A, Klimek L, Mösges R: The conjunctival provocation test (CPT): Reproducibility and prospective validation in a clinical trial. J Allergy Clin Immunol, submitted. 